\title{
Incorporation of Impacted Morselized Bone Allografts in Rabbits
}

\author{
F. Judas, M.H. Figueiredo, A.M.S. Cabrita, and A. Proença
}

\begin{abstract}
Morselized bone allografts have been used for the treatment of bone stock loss in orthopedic revision surgery with encouraging results. However, several parameters can influence the graft incorporation including the processing treatments. This experimental work used a cavitary bone defect in 90 rabbits to evaluate the sequence of incorporation of three different kinds of morselized bone allografts: uncryopreserved cancellous bone, freeze-dried cancellous bone, and totally demineralized cortical bone each of which were prepared in accordance with our rigid protocol. Revascularization and remodeling of the transplanted bone grafts were evident upon histological evaluation. Bone apposition and bone resorption resulted in a mixture of graft and new bone. Mineralized cancellous grafts showed great osteoconductive capacity, whereas demineralized cortical grafts showed an intense osteoinductive capacity and a weak osteoconductive capacity. In a general evaluation, cryopreserved cancellous bone grafts showed superior biological efficacy for reconstruction of experimental bone defects, closely followed by freeze-dried cancellous bone grafts, and, finally, by demineralized cortical bone grafts. These results validate our protocol for the processing and preservation of these three kinds of bone grafts.
\end{abstract}

$\mathrm{B}$ ONE STOCK LOSS is the main problem for revision surgery of a failed total hip prosthesis. In our institution, impacted morselized bone allografts are frequently used in conjunction with a roof reinforcement ring for acetabular reconstruction and a cementless stem on the femoral side, even in large cavitary defects. Initially, these defects were filled with bone cement but it soon became clear that the failure rate of this technique was unacceptably high. Although the reconstruction of the osteolyses induced by aseptic loosening of hip prosthesis with impacted morselized cancellous allografts had demonstrated satisfactory results, ${ }^{1-4}$ controversy still exists about the best approach to these bone stock deficiencies. It is recognized that, in addition to the surgical technique, the methods of bone allograft processing, preservation, and sterilization can alter both its initial physical and chemical properties and their immune response, as well as endanger the mechanical stability of the surgical reconstruction, ${ }^{5}$ concomitantly influencing the biological behavior of the allografts.

This study evaluated the incorporation sequence of three different kinds of morselized bone allografts-cryopreserved cancellous bone, freeze-dried cancellous bone, and totally demineralized cortical bone-prepared in accordance with our current protocol. An experimental model was developed using a cavitary bone defect in rabbits. The impacted bone allografts were placed into the cancellous bone of the rabbit knee under mechanical stability conditions.

\section{MATERIALS AND METHODS \\ Preparation of Graft Material}

All bone grafts were harvested from donor Californian rabbits using standard aseptic techniques. The cancellous bone was harvested from the femoral and tibial condyles, treated in $70 \%$ ethanol and hydrogen peroxide solutions, and stored in liquid nitrogen for at least 2 weeks. The freeze-dried allografts underwent a complementary sterilization procedure using gamma rays (25-kGy dose). The cortical diaphyseal femoral bone was demineralized at room temperature in a $2.4 \mathrm{~N} \mathrm{HCl}$ solution (for 48 hours), defatted in a hexachlophene-ethanol solution, and preserved in a $0.5 \%$ formaldehyde solution at $4^{\circ} \mathrm{C}$. The grafts were cut into small bone chips (morselization) yielding cancellous chips of size 200 to $400 \mu \mathrm{m}$, and cortical chips of size 700 to $800 \mu \mathrm{m}$.

From the Orthopaedics Department of the Coimbra University Hospitals (F.J., A.P.), and Center of Histophysiology, Experimental Pathology and Developmental Biology-Faculty of Medicine of the University of Coimbra (M.H.F., A.M.S.C.), Coimbra, Portugal.

Address reprint requests to Fernando Judas, MD, $\mathrm{PhD}$, Orthopaedics Department of Coimbra University Hospitals (HUC), Praceta Prof Mota Pinto, Bloco de Celas, 3000 Coimbra, Portugal. E-mail: fernandojudas@iol.pt

(c) 2005 by Elsevier Inc. All rights reserved. 360 Park Avenue South, New York, NY 10010-1710 


\section{Grafting Method}

Ninety Californian 9-month-old male rabbits (of body weight 3400 $\pm 0039 \mathrm{~kg}$ ) underwent graft impaction into a bone defect (diameter, $6 \mathrm{~mm}$; depth, $14 \mathrm{~mm}$ ) in the medial condyle of the right femur, under general anesthesia and aseptic conditions. The opposite side was used for the control. Animals were divided into three groups of 30 animals each: group I-cryopreserved cancellous bone; group II-freeze-dried cancellous bone; and group III-totally demineralized cortical bone. The animals in each group were subgrouped as 10 animals, sacrificed at the end of anesthesia and day 15 (subgroup A); on day 30 (subgroup B); or on day 60 (subgroup C). Animals were maintained according to the laws of animal welfare.

The distal extremity of the femur was submitted to histological evaluation, using the methods developed by Schenk, ${ }^{6}$ of hematoxylin-eosin and of trichrome Masson staining. Statistical analyses were performed with Student $t$ test, using a commercial software package (Excel program, Statview 4.1).

\section{RESULTS}

The results were evaluated as a function of the postoperative time: 15, 30 or 60 days. Histomorphological studies of both decalcified and undecalcified material showed the revascularization process and apposition of new host bone on the fragments of the three kinds of grafts. None of the grafts showed the presence of a fibrosis capsule or biological intolerance reactions. The three kinds of bone grafts were incorporated. New bone formed by creeping substitution of bone grafts as well as endochondral and intramembranous processes. Intramembranous ossification was the main mechanism of osteogenesis in all groups. On the other hand, neither collection nor infiltration of lymphocytes, or of small round cells, was found in any of the defects filled with the bone grafts. In contrast control defects showed bone formation with great amount of fibrous tissue at postoperative day 60 .

Cryopreserved cancellous bone grafts were almost totally revascularized and replaced by new host living bone. Freeze-dried cancellous bone grafts showed similar biological behavior to cryopreserved cancellous bone grafts. However, the former showed a slower, less complete incorporation. A less extensive, more prolonged process of incorporation was observed in demineralized cortical bone grafts compared to mineralized cancellous bone grafts. Mineralized cancellous grafts showed great osteoconductive capacity, whereas demineralized cortical grafts revealed an intense osteoinductive capacity and a weak osteoconductive capacity.

In a general evaluation, cryopreserved cancellous bone grafts showed superior biological efficacy for reconstruction of an experimental bone defect, closely followed by freezedried cancellous bone grafts, and, finally, by demineralized cortical bone grafts. In all groups, the biological events related to the incorporation of bone allografts were not complete at the end of the experimentation. The demineralized cortical bone graft group showed the presence of numerous incompletely remodeled graft fragments.

\section{DISCUSSION}

The validity of the impaction allografting technique has been demonstrated previously by histological and mechanical criteria. Histologically, grafted bone chips were shown to be replaced by host bone in human retrieval studies and animal experiments. The mechanical properties of morselized cancellous allografts themselves have also been reported. ${ }^{3,4}$ On the other hand, processing treatments are paramount to incorporation of the bone grafts. ${ }^{1}$

The three kinds of bone grafts used in this experimental study were revascularized and incorporated, meaning that the grafts were surrounded by living tissue, with new bone formation on the graft, which does not necessarily have to be totally resorbed. Mineralized cancellous grafts showed great osteoconductive capacity, whereas demineralized cortical grafts presented an intense osteoinductive capacity and a weak osteoconductive capacity. Previous studies have been shown that mineralized cancellous bone graft, as compared to demineralized cortical bone, not only reveal a higher porosity but also a wider pore size distribution, which is much more favourable for early bone regeneration. ${ }^{8}$ On the other hand, the production of a large fracture surface area, by microfracturing the bone during morselization, they expose the bone matrix to surrounding tissues ${ }^{9}$ and the impaction technique release bone growth factors from the cancellous bone graft.

Urist and Peltier ${ }^{10}$ demonstrated that demineralized bone matrix placed into subcutaneous or intramuscular sites induced new bone formation. Processing methods for this matrix influence its osteoinductive performance. Hydrochloric acid $(0.5$ to $0.6 \mathrm{~N})$ is the most commonly used demineralizing agent. ${ }^{11-13}$ In this study, $2.4 \mathrm{~N} \mathrm{HCl}$ solution did not influence the DBM osteoinductive properties in a orthotropic situation.

On the other hand, no collection or infiltration of lymphocytes, or of small round cells, characteristic of an immune response, was observed in any defect filled with bone grafts, leading to the assumption that the phenomena of graft rejection did not influence the biological behavior of bone grafts. Neither surviving cells nor bone marrow were present in these grafts. The major source of antigens in a bone graft is the cellular elements. ${ }^{14}$

One limitation of this study is that there was no direct loading of grafts. However, the sequence of histological events that occurred in our specimens was identical to that observed in the process of incorporation of impacted morselized allografts in loaded animal studies. Schreurs et $\mathrm{al}^{15}$ reported that loads play a minor role during the initial phase of graft incorporation. Other factors related to the trauma induced by surgery or the immunological conditions are much more important.

Histomorphological findings of this study showed cryopreserved morselized cancellous bone grafts to have a superior biological efficacy for reconstruction of experimental bone defects, closely followed by freeze-dried morselized cancellous bone grafts and, finally, by demineralized 
morselized bone grafts. These results validate our protocol for the processing and preservation of these three kinds of bone grafts. ${ }^{7}$

\section{REFERENCES}

1. Proença A, Judas F, Cabral R, et al: Revision surgery of hip prosthesis. Osteolysis reconstruction with bone allografts. Orthopaedics Department of Coimbra University Hospitals, 1996

2. Proença A: "Conelock" stem in femoral revision. XI International Course of Osteo-articular Reconstruction Surgery, Madrid, 2002, p 82

3. Slooff TJJH, Schreurs BW, Gardeniers JWM, et al: Revision total hip arthroplasty with impacted cancellous allografts and cement. In Pipino F (ed): Bone Cement and Cemented Fixation of Implants 40 Years of Clinical Practice and Prospective for the future. 2001, p 163

4. Gie GA, Ling RSM: Contained, morsellised, impacted allograft in revision total hip arthroplasty, the femoral side. Orthopaedics Today 1:18, 1998

5. Tomford WW, Mankin HJ: Bone banking. Update on methods and materials. Orthop Clin N Am 30:565, 1999
6. Schenk RK: Preparation of calcified tissues for light microscopy. In Methods of Calcified Tissues Preparation, Vol. 1, London: Elsevier Science Publishers; 1984.

7. Boyce T, Edwards J, Scarborough N: Allograft bone. The influence of processing on safety and performance. Orthop Clin $\mathrm{N}$ Am 30:571, 1999

8. Judas F, Proença A, Moura M, et al: The relevance of physical characterization in the evaluation of performance of bone allografts. Key Engineering Materials 230-232:467, 2002

9. Tägil M, Aspenberg P: Impaction of cancellous bone grafts impairs osteoconduction in titanium chambers. Clin Orthop 352: 231, 1998

10. Urist MR, Peltier LF: The classic: bone: formation by autoinduction. Clin Orthop 395:4, 2002

11. Judas F: Contribution to the study of Morselized Bone Allografts and Biomaterials. PhD Thesis. Coimbra University, 2002

12. Proença A, Judas F, Canha N: Bone banking organization. Rev Ortop Traum 12:171, 1986

13. Proença A: Bone and osteochondral allografts. PhD Thesis. Coimbra University, 1990

14. Bauer TW, Muschler GF: Bone graft materials. An overview of the basic science. Clin Orthop 371:10, 2000

15. Schreus BW, Slooff TJJH, Buma P, et al: Basic science of bone impacting grafting. AAOS Instructional Course Lectures Vol 50:211, 2001 